Article type : Original

Manuscript number: HEP-18-0074

\title{
ADAPT: An algorithm incorporating PRO-C3 accurately identifies patients with NAFLD and advanced fibrosis
}

Samuel J. Daniels ${ }^{1,2}$, Diana J. Leeming ${ }^{1}$, Mohammed Eslam ${ }^{3}$, Ahmed M. Hashem ${ }^{4}$, Mette J. Nielsen ${ }^{1}$, Aleksander Krag ${ }^{2,5}$, Morten A. Karsdal ${ }^{1}$, Jane I. Grove ${ }^{6,7}$, Indra Neil Guha ${ }^{6,7}$, Takumi Kawaguchi ${ }^{8}$, Takuji Torimura $^{8}$, Duncan McLeod ${ }^{9}$, Jun Akiba ${ }^{10}$, Philip Kaye ${ }^{11}$, Bastiaan de Boer ${ }^{12}$, Guruprasad P. Aithal ${ }^{6,7^{*}}$, Leon A. Adams ${ }^{13^{*}}$, Jacob George ${ }^{3^{*}}$

${ }^{1}$ Nordic Bioscience Biomarkers and Research A/S, Herlev, Denmark

${ }^{2}$ Department of Gastroenterology and Hepatology, Odense University Hospital, Odense, Denmark

${ }^{3}$ Storr Liver Centre, The Westmead Institute for Medical Research, University of Sydney and Westmead Hospital, Westmead, Australia

${ }^{4}$ Department of Systems and Biomedical Engineering, Faculty of Engineering, Minia University, Minia, Egypt

${ }^{5}$ Institute of Clinical Research, University of Southern Denmark, Odense, Denmark

${ }^{6}$ Nottingham Digestive Diseases Centre, The University of Nottingham, Nottingham NG7 2UH.

${ }^{7}$ NIHR Nottingham Biomedical Research Centre at the Nottingham University Hospitals NHS Trust and the University of Nottingham, Nottingham NG7 2UH

${ }^{8}$ Division of, Department of Medicine, Kurume University School of Medicine, Kurume, Japan

${ }^{9}$ Department of Anatomical Pathology, Institute of Clinical Pathology and Medical Research (ICPMR), Westmead Hospital, Sydney, Australia

${ }^{10}$ Department of Diagnostic Pathology, Kurume University Hospital, Kurume, Japan.

${ }^{12}$ Department of Anatomical Pathology, PathWest, Fiona Stanley Hospital

${ }^{13}$ Medical School, The University of Western Australia, Nedlands, Australia

*Share joint last authorship

This article has been accepted for publication and undergone full peer review but has not been through the copyediting, typesetting, pagination and proofreading process, which may lead to differences between this version and the Version of Record. Please cite this article as doi: 10.1002/hep.30163

This article is protected by copyright. All rights reserved. 
Email: SJD sjd@nordicbio.com, DJL djl@nordicbio.com, ME mohammed.eslam@sydney.edu.au, AMH ahmedhashim81@gmail.com, MJU mju@nordicbio.com, AK Aleksander.Krag@rsyd.dk, MAK mk@nordicbio.com, JIG Jane.Grove@nottingham.ac.uk, ING Neil.Guha@nottingham.ac.uk, TK takumi@med.kurume-u.ac.jp, TT tori@med.kurume-u.ac.jp, DM

Duncan.McLeod@health.nsw.gov.au, JA akiba@med.kurume-u.ac.jp, KP

Philip.Kaye@nuh.nhs.uk; BDB bastiaan.deboer@health.wa.gov.au, GPA

Guru.Aithal@nottingham.ac.uk, LAA leon.adams@uwa.edu.au, JG jacob.george@sydney.edu.au

Corresponding author: Samuel J. Daniels, Nordic Bioscience, Herlev Hovedgade 205-207, 2730

Herlev, Denmark Phone:+45 44525252 Fax: +45 44525251 email: sjd@nordicbio.com

\section{List of Abbreviations}

ADAMTS: A disintegrin and metalloproteinase with thrombospondin motifs

ALT: Alanine Aminotransferase

APRI: AST to Platelet Ratio Index

AST: Aspartate Aminotransferase

AUROC: Area under receiver operating curve

BMI: Body mass index

ECM: Extracellular matrix

ELISA: Enzyme-linked immunosorbent assay

GGT: Gamma-Glutamyltransferase

HDL: High-density lipoprotein

LDL: Low-density lipoprotein

LHR+: Positive likelihood ratio

LHR-: Negative likelihood ratio

MS: Metabolic syndrome

NAFL: Non-alcoholic fatty liver

NAFLD: Non-alcoholic fatty liver disease

NASH: Non-alcoholic steatohepatitis

NFS: NAFLD Fibrosis Score

NPV: Negative predictive value

This article is protected by copyright. All rights reserved. 
PPV: Positive predictive value

\section{Funding:}

We acknowledge the Danish Agency for Science, Technology and Innovation and the Danish National Research Foundation supporting this work.

\section{Abstract}

Background and Aim: Given the high global prevalence of non-alcoholic fatty liver disease (NAFLD), the need for relevant non-invasive biomarkers and algorithms to accurately stage disease severity is a critical unmet medical need. Identifying those with advanced fibrosis $(\geq F 3)$ is the most crucial, as these individuals have the greatest risk of adverse, long-term, liver-related outcomes. We aimed to investigate the role of PRO-C3 (a marker of type III collagen formation) as a biomarker for advanced fibrosis in NAFLD. Methods: We measured PRO-C3 by enzyme-linked immunosorbent assay (ELISA) in two large independent cohorts with extensive clinical phenotyping and liver biopsy; 150 in the derivation and 281 in the validation cohort. A PRO-C3 based fibrosis algorithm that included Age, presence of DiAbetes, PRO-C3 (a marker of type III collagen formation), and plaTelet count ("ADAPT") was developed. Results: PRO-C3 increased with fibrosis stage (rho $0.50 \mathrm{p}<0.0001$ ) and was independently associated with advanced fibrosis ( $\mathrm{OR}=1.05,95 \% \mathrm{Cl} 1.02-1.08, \mathrm{p}=0.003$ ). ADAPT showed areas under the receiver operating characteristics curve (AUROC) of 0.86 (95\% $\mathrm{Cl} 0.79$ to 0.91 ) in the derivation and 0.87 in the validation cohort ( $95 \% \mathrm{Cl} 0.83$ to 0.91$)$ for advanced fibrosis. This was superior to the existing fibrosis scores, aspartate aminotransferase (AST) to platelet ratio index (APRI), FIB-4 and NAFLD fibrosis score (NFS) in most comparisons. Conclusion: PRO-C3 is an independent predictor of fibrosis stage in NAFLD. A PRO-C3 based score (ADAPT) accurately identifies patients with NAFLD and advanced fibrosis and is superior to APRI, FIB-4 and NFS.

This article is protected by copyright. All rights reserved. 


\section{Keywords}

Biomarker, Extracellular matrix, Non-invasive score, Non-alcoholic fatty liver disease, PRO-C3

\section{Introduction}

The increase in global prevalence of metabolic syndrome (MS) has been accompanied by a rise in organ damage including end stage disease related to non-alcoholic fatty liver disease (NAFLD). Estimates place the worldwide prevalence of NAFLD at $25 \%{ }^{1}$. A subset of these patients develop non-alcoholic steatohepatitis (NASH) that can progress to cirrhosis and are at a high risk of adverse liver-related outcomes ${ }^{1}$. From a management and therapeutic perspective, an unmet clinical need is the requirement to distinguish those with early disease from those at highest risk of clinical complications. While metabolic hepatic inflammation is the milieu that drives disease progression, various studies (including meta analyses) that have examined for prognostic histological features suggest that fibrosis stage is the parameter that best associates with overall- and liver-related mortality, as well as liver transplantation and liver related events ${ }^{2-5}$.

The gold standard for the evaluation of liver fibrosis stage is percutaneous needle biopsy, which is compromised by inherent sampling and inter-observer biases and peri-procedural risk ${ }^{6,7}$. The invasiveness and costs of performing biopsies also makes it unsuitable for mass screening, for staging and risk stratification. The latter is important as the majority of patients with advanced fibrosis and even cirrhosis, are asymptomatic and often indistinguishable from those at earlier disease stages ${ }^{8,9}$. In this context, there is a need for surrogate markers of disease stage that can identify and risk stratify patients with NAFLD. This area of research can broadly be divided into liquid (typically blood based) or physical approaches (measurement of liver stiffness). Physical approaches while promising are less useful for population level screening and are limited by cost and other technique-specific considerations. Several serum based biomarker tests have previously been developed and applied to NAFLD patients ${ }^{10-14}$. These scores typically combine clinical features and

This article is protected by copyright. All rights reserved. 
routine laboratory tests and are used primarily to rule out advanced fibrosis ${ }^{15}$, however they lack sufficient diagnostic accuracy and sensitivity.

Liver fibrosis is characterized by the accumulation of excess extracellular matrix (ECM) and hence biomarkers reflecting structural changes occurring in the hepatic ECM during chronic injury may be of value in the assessment of fibrosis progression or regression. We recently demonstrated that PRO-C3, an ADAMTS generated neo-epitope marker of type III collagen formation, is a marker of fibrosis in patients with chronic hepatitis C. The role of PRO-C3 in patients with NAFLD however, is largely unknown. Since the performance of biomarkers and non-invasive liver fibrosis scores varies widely according to disease etiology, whether PRO-C3 has a role as a biomarker in NAFLD is unclear. In this study, we sought to a) explore the association of PRO-C3 with liver fibrosis in two large independent biopsy-proven cohorts with NAFLD and b) determine if PRO-C3 can be combined with simple and routinely available clinical variables in to a novel score for the prediction of advanced fibrosis in patients with NAFLD. We compared the performance of our derived model with other known biomarker algorithms.

\section{Materials and Methods}

\section{Study population}

A total of 431 well phenotyped patients with biopsy confirmed NAFLD comprised the study cohort. The derivation cohort included 150 patients from the Storr Liver Centre, Sydney, Australia; the validation cohort comprised 281 patients recruited from four international sites, Nottingham University Hospitals NHS Trust, United Kingdom ( $n=42)$; Kurume University School of Medicine, Kurume, Japan ( $n=48)$; University of Western Australia, Nedlands, Australia ( $n=144)$ and 47 additional patients from the Storr Liver Centre.

All patients were referred for the investigation of abnormal liver tests or steatosis detected by

This article is protected by copyright. All rights reserved. 
ultrasound. The diagnosis of NAFLD was established by liver biopsy in all cases. Patients with disease of other etiologies including viral hepatitis and auto-immune liver disease were excluded by standard clinical, laboratory and histopathological assessments. Patients with evidence of hepatic decompensation, secondary causes of steatosis, including excess alcohol (men, $>30 \mathrm{~g} / \mathrm{day}$; women, $>20 \mathrm{~g} /$ day), total parenteral nutrition or the use of drugs known to precipitate steatosis were excluded.

Demographic and clinical data were obtained, including age, gender, ethnicity, height, weight, and waist circumference at the time of biopsy. Body mass index (BMI) was calculated as $\mathrm{BMI}=\mathrm{kg} / \mathrm{m}^{2}$. Arterial hypertension was defined as blood pressure $\geq 130 / \geq 85 \mathrm{mmHg}$ or treatment with antihypertensive drugs. Diabetes was defined as a fasting blood glucose $\geq 7.0 \mathrm{mmol} / \mathrm{L}$, previous diagnosis of diabetes or use of anti-diabetic drugs. Hyperlipidemia was defined as fasting total cholesterol $>5.5 \mathrm{mmol} / \mathrm{L}$, triglycerides $>1.7 \mathrm{mmol} / \mathrm{L}$ or treatment with lipid-lowering drugs. Ethical approval and written informed consent from patients was obtained from all participating centers.

\section{Histology}

All biopsies were routinely stained with hematoxylin \& eosin and Masson's Trichrome. The stained sections were read and scored by an expert liver pathologist at each participating center using the scoring system proposed by Kleiner et al., $2005^{16}$. The stage of liver fibrosis was defined as: stage 0 , absence of fibrosis; stage 1 , perisinusoidal or portal fibrosis; stage 2 perisinusoidal and portal/periportal fibrosis; stage 3 septal or bridging fibrosis; and stage 4 as cirrhosis. A diagnosis of NASH was according to the EASL-EASD-EASO guidelines. ${ }^{17}$ Thirty-one biopsies were scored independently by pathologists from the various centers, and inter observer agreement was calculated using the $\mathrm{K}$ statistic and was $=0.55$ for fibrosis, comparable to previously published results $^{18-21}$.

This article is protected by copyright. All rights reserved. 


\section{Biomarker quantification}

At the time of biopsy, a fasting blood sample was obtained and routine biochemical tests were performed using standard methods and assays. Biochemical tests included albumin, alanine aminotransferase (ALT), aspartate aminotransferase (AST), total cholesterol, gammaglutamyltransferase (GGT), insulin, high-density lipoprotein (HDL), low-density lipoprotein (LDL), platelets, and triglycerides. Additional blood samples were drawn and frozen at $-80^{\circ} \mathrm{C}$ for future research. Type III collagen formation was assessed in serum using the PRO-C3 competitive ELISA assay from Nordic Bioscience, Herlev, Denmark, as previously described ${ }^{22}$.

The APRI, FIB-4 and NAFLD Fibrosis Scores were calculated using clinical and routine laboratory variables and previously defined algorithms and cut-off values for NAFLD/NASH patients ${ }^{10,12,14,23}$.

\section{Statistical analysis}

The main aim of this study was the development of an algorithm comprised of clinical and laboratory variables that could accurately distinguish patients with advanced fibrosis $(F \geq 3)$ from those without. To this end, patients in the derivation cohort were stratified into those with advanced fibrosis $(\mathrm{F} \geq 3)$ and those without (FO-2). Stage 2 and 3 for lobular and portal inflammation was pooled as only 1 patient in both groups was graded stage 3 . Continuous variables in the two groups were compared using the $t$ test and categorical variables were compared using Fisher's exact test. Comparisons between mean marker levels were performed using the Kruskal-Wallis test followed by Dunn's multiple comparison test. Variables that were significantly different between patients with advanced fibrosis and those without advanced fibrosis were identified as potential algorithm components.

For the formulation of predictive models, variables showing a $\mathrm{p}<0.05$ at univariate analysis (Student $t$ test for parametric variables, and $\mathrm{X}^{2}$ or Fisher exact test for frequencies) were included. The interaction between these variables was first tested. Variables explaining a statistically significant proportion of the variance $(p<0.05)$ were maintained in the model using the likelihood ratio $(L R)$

This article is protected by copyright. All rights reserved. 
test. The model variables were selected using the leave-one-out method to facilitate the calculation of over-fit bias reduced estimates ${ }^{24}$. To avoid over-fitting, 10 -fold cross validations were used in the tree building process.

The model was as following:

$$
A D A P T=\exp \left(\log _{10}\left(\frac{\text { Age } \times \text { PRO-C3 }}{\sqrt{\text { Platelets }}}\right)\right)+\text { Diabetes }
$$

The discriminative ability of the model for the identification of severe fibrosis $(F \geq 3)$ was assessed by means of receiver operating characteristic curve analysis and expressed as area under the receiver operating characteristic curve (AUROC). A cut-off value to distinguish patients with advanced fibrosis from those without was determined using the bootstrap Youden Index. The diagnostic accuracy of the algorithm and the derived cut-off was determined by calculating sensitivity, specificity, positive predictive value (PPV) and negative predictive value (NPV). To overcome both spectrum effect and ordinal scale issues, we undertook two approaches. Firstly, we used the Obuchowski measure, as proposed by Lambert et $a^{25,26}$, which is a measure of the probability that two randomly chosen patients from different fibrosis stages are correctly classified according to the weighted scheme, with a penalty for incorrect classification. In the second method, we standardized the AUROC for the distribution of fibrosis stages as proposed by Poynard et $\left.a\right|^{27}$, as recently described ${ }^{28}$.

ROC curves were also calculated for the established diagnostic scores, APRI, FIB-4 and NAFLD Fibrosis Score. Sensitivity, specificity, positive predictive value (PPV), negative predictive value (NPV), positive and negative likelihood ratio $\left(\mathrm{LHR}^{+}, \mathrm{LHR}^{-}\right)$and $95 \%$ Cls were calculated. Estimates of AUROCs and comparisons between AUROCs were performed using the method suggested by Hanley and $\mathrm{McNeil}^{29}$. Validation was subsequently performed on the validation cohort as well as for the combined overall cohort.

This article is protected by copyright. All rights reserved. 
All data are shown as medians and variation expressed via Tukey plots. P-values $<5 \%$ were considered significant. Model building and statistical analysis was performed using MedCalc version 16.8.4 (MedCalc Software, Ostend, Belgium) and SAS version 9.1 (SAS Institute Inc., Cary, NC, USA). Graphs were designed using GraphPad Prism version 7 (GraphPad Software, Inc., CA, USA).

\section{Results}

\section{Patient Characteristics}

The characteristics of the 150 NAFLD patients used to develop the model (derivation cohort) and the 281 used to test the model (validation cohort) are shown in Table 1. Serum levels of albumin, AST, cholesterol and HDL were all significantly lower in the validation cohort when compared to the derivation cohort. In addition, both BMI and insulin level were found to be significantly elevated. No other significant differences were observed between the two cohorts. The prevalence of severe fibrosis was not significantly different between the cohorts.

PRO-C3 is highly associated with severity of fibrosis and histological parameters

Within the derivation cohort, a neo-epitope marker of type III collagen formation, PRO-C3, was found to be significantly elevated in patients with advanced fibrosis $(F \geq 3)$ compared to the mild/moderate group ( $p<0.0001)$. PRO-C3 was highly associated with disease severity (Figure 1) and moderately correlated to the severity of fibrosis $(r h o=0.501, p<0.0001)$. PRO-C3 was able to discriminate between the following stages of fibrosis (Figure 1): F0 versus F2 (27\% increase, $p<0.0332)$, F0 versus F3 (54\% increase, $p<0.0001), F 1$ versus F3 ( $36 \%$ increase, $p<0.0002$ ) and F0 versus F4 (57\% increase, $\mathrm{p}<0.0021)$. In addition, PRO-C3 discriminated between the various stages of hepatocyte ballooning (stage 0 versus stage $1 p=0.001$, stage 0 versus $2 p=0.0003$ ), lobular inflammation (stage 0 versus stage $1 p=0.0004$, stage 0 versus stage 2 and $3 p=0.0008$ ) and steatosis (stage 1 versus stage $3 p=0.003$ ).

This article is protected by copyright. All rights reserved. 
We undertook logistic regression to discern the effect of various clinical variables on the association of $\mathrm{PRO}-\mathrm{C} 3$ with the presence of advanced fibrosis $(\mathrm{F} \geq 3)$ within the derivation cohort. In this analysis, PRO-C3, when adjusted for age, ALT, AST, BMI, ballooning, lobular inflammation, presence of diabetes, GGT and platelet count, was independently associated with advanced fibrosis ( $O R=1.054$, 95\% $\mathrm{Cl}$ 1.01-1.07) (Table 2). The area under the receiver-operating curve (AUROC) for the identification of patients with advanced fibrosis $(\mathrm{F} \geq 3$ ) of $\mathrm{PRO}-\mathrm{C} 3$ alone was 0.81 (95\% $\mathrm{Cl}$ 0.74-0.87) (Data not shown).

\section{Clinical parameters associated with the level of PRO-C3}

Given that univariate and multivariate analyses revealed that the level of PRO-C3 was a strong predictor of advanced fibrosis, we examined for clinical parameters associated with the level of PROC3. It was subsequently found that ALT (rho 0.29, p=0.0004), AST (rho 0.42, $p<0.0001$ ), fasting blood glucose (rho 0.23, $p=0.007$ ), insulin level (rho 0.43, $p<0.0001$ ), platelet count ( $r$ ho $-0.24, p=0.004$ ) and presence of diabetes (rho 0.16, $\mathrm{p}=0.05$ ) all correlated with the level of PRO-C3 to varying degrees.

Development of a PRO-C3 based predictive fibrosis score (ADAPT)

Based on the finding that PRO-C3 is strongly associated with fibrosis, we sought to build a model for the prediction of significant fibrosis based on PRO-C3 and routinely assessed clinical and laboratory variables. Patients within the derivation cohort were divided into two groups according to NASH CRN fibrosis stage, FO-2 (no fibrosis to moderate fibrosis) and F3-4 (advanced fibrosis) (Table 3). PRO-C3 was elevated in patients with advanced fibrosis compared to the mild to moderate group $(p<0.0001)$. Furthermore, those with advanced fibrosis had significantly increased levels of AST, GGT and a higher AST/ALT ratio (Table 3). As would be expected, patients with advanced fibrosis had a worse metabolic profile with lower LDL, higher circulating insulin levels and a higher waist-to-hip ratio (Table 3). The presence of diabetes was more likely in patients with advanced fibrosis; $67 \%$ of

This article is protected by copyright. All rights reserved. 
patients with F3-4 had diabetes compared to just $29 \%$ of the F0-2 group ( $p=0.002$ ) (Table 3 ). In addition, patients with advanced fibrosis were found to be older $(p=0.02)$ and with a lower platelet count compared to those without $(p=0.002)$ (Table 3).

Variables that were significantly different between the two groups $(p<0.05)$ were considered eligible for the model building process. Those that described a statistically significant proportion of the variance were included in the model using the likelihood ratio (LR) test. Ultimately, the variables that were included within the model, named "ADAPT", were age, presence of diabetes, platelet count and PRO-C3.

The diagnostic capability of the ADAPT score was assessed via AUROC and was higher than that of PRO-C3 alone, yielding an AUROC of $0.86(95 \% \mathrm{Cl} 0.79-0.91)$ (Figure 2).

Validation of the diagnostic capabilities of the ADAPT score

To ascertain the validity of our model, the ability of ADAPT to identify patients with advanced fibrosis was corroborated in a separate cohort comprised of patients from four centers across AsiaPacific and Europe $(n=281)$. Several significant differences were identified between the derivation and the validation cohort; these differences reflect the heterogeneity of NAFLD patients with advanced fibrosis. Despite cohort differences, the diagnostic accuracy of ADAPT was maintained with an AUROC in the validation cohort of 0.87 (95\% Cl 0.83-0.91) (Figure 3).

The diagnostic performance of a score, when assessed by AUROC, may vary according to disease prevalence, known as spectrum bias ${ }^{30}$. The Obuchowski measure accounts for the spectrum bias and provides a means by which the diagnostic accuracy of a score can be assessed. The Obuchowski measure of ADAPT within the derivation cohort was calculated to be 0.86 and within the validation cohort it was 0.89 . Additionally, we standardized the AUROC for the distribution of fibrosis stages according to Poynard et $a l^{27}$. The standardized AUROC of ADAPT was found to be 0.89 and 0.89 within the derivation and validation cohorts, respectively (Table 4). For further confirmation of the generalizability of the model, the validation cohort was stratified into various groups according to

This article is protected by copyright. All rights reserved. 
age, $(<50,50-60$ and $>60), \mathrm{BMI}$, Sex, NASH vs NAFL and center. In this analysis, ADAPT remained a robust algorithm in that the AUROC was maintained across all sub-populations, with NPV consistently exceeding 90\% (Supplementary Figure 1).

\section{Performance of ADAPT against standard algorithms}

Within the derivation cohort the AUROC of "ADAPT" (AUROC=0.855) was superior to clinically available serum based non-invasive scores: APRI (AUROC $=0.73, p=0.02$ ), FIB-4 (AUROC=0.78, $p=0.06$ ) and NAFLD Fibrosis Score (AUROC=0.78, $p=0.06$ ) (Table 4). Likewise, in the validation cohort, the AUROC of "ADAPT" (AUROC=0.87) was greater than APRI (AUROC=0.78, $p=0.0005$ ), FIB-4 (AUROC $=0.85, p=0.32$ ) and NAFLD Fibrosis Score (AUROC=0.79, $p=0.02$ ) (Table 4). Adjusting the AUROC according to Poynard et $\left.a\right|^{27}$ caused minor increases in the AUROC in all scores (Table 4). Further investigation into the ability of ADAPT to identify patients with clinically significant fibrosis (F2-F4) highlighted the superiority of the ADAPT score when compared to other clinically available serum based non-invasive scores (supplementary table 3).

\section{Derivation of cut-off values}

The derivation cohort was subjected to ROC curve analysis to derive a cut-off value for the rule-in and rule-out of advanced fibrosis. A value of $>6.3287$ for the rule in/out of advanced fibrosis was identified by the Youden Index, PPV 48.4\%, NPV 96.6\%, (Supplementary table 2). By applying this cut-off, 73\% ( $n=158$ ) F0-2 patients were correctly classified and 27\% ( $n=58)$ incorrectly classified. Among F3-4 patients, 92\% ( $n=60)$ were correctly classified while $8 \%(n=5)$ were incorrectly classified (Table 5). We applied previously derived cut-off values for APRI (rule in advanced fibrosis $>1.5$, rule out advanced fibrosis $<0.5$ ), FIB-4 (rule in advanced fibrosis $>2.67$, rule out advanced fibrosis $<1.3$ ) and NAFLD Fibrosis Score (rule in advanced fibrosis $>0.676$, rule out advanced fibrosis $<-1.455)^{10,14,23}$. A large proportion of patients fell within an indeterminate zone, table 5. FIB-4 and APRI showed reasonable performance at identifying patients without advanced liver fibrosis, $68 \%(n=147)$ and

This article is protected by copyright. All rights reserved. 
$67 \%(n=145)$ were correctly classified, respectively. However, these scores performed poorly at identifying patients with advanced liver fibrosis, NAFLD Fibrosis Score and FIB-4 correctly identified $51 \%(n=33)$ and $46 \%(n=30)$ patients, respectively.

\section{Discussion}

In this study, we measured PRO-C3 in NAFLD patients from centers across the world and with a wide variation in ages and clinical manifestations, similar to that observed in daily clinical practice. The principal findings were that: 1) PRO-C3 progressively increases with fibrosis severity in NAFLD but that the association remains highly significant even after adjustment for multiple biochemical and clinical parameters, and 2) PRO-C3 when combined with routine clinical parameters (ADAPT) generated a highly accurate tool for the detection of advanced fibrosis in NAFLD. ADAPT is thus a unique score that has utility for risk stratification and for the clinical management of patients with non-alcoholic fatty liver disease.

Non-invasive biomarkers that reflect the process of hepatic fibrosis are urgently needed; collagen formation biomarkers are thus attractive targets. Here we demonstrate that PRO-C3, which measures type III collagen synthesis is a novel and precise marker for advanced liver fibrosis in concordance with what we have recently shown in chronic hepatitis $C^{22,31,32}$. Notably, a recent small non-biopsy study ( $n=297$ ) from a phase III study of balaglitazone in patients with late-stage Type 2 diabetes (BALLET study) suggested that PRO-C3 could have utility as a determinant of treatment response to a potential anti-fibrotic therapy ${ }^{33}$. Karsdal et al (2016) subsequently confirmed this within a study investigating the anti-fibrotic efficacy of farglitazar ${ }^{33}$; Harrison et al (2018) further explored PRO-C3 as a determinant of treatment response within a phase IIb study ${ }^{34}$. Though that finding needs to be validated in biopsy proven cohorts, their data in combination with our findings suggest that PRO-C3 could serve as a biomarker not only for prediction of fibrosis progression, but also for treatment response. Interestingly, the optimal cut off value in our study was $15.6 \mathrm{ng} / \mathrm{ml}$ for

This article is protected by copyright. All rights reserved. 
advanced fibrosis, which is significantly different from that in patients with hepatitis $C(20 \mathrm{ng} / \mathrm{ml})^{35}$. Consistently, the cut off level for PRO-C3 was also lower in the BALLET report $(13.1 \mathrm{ng} / \mathrm{ml})^{33}$. Further studies will be required to confirm the optimal cut off in NAFLD. It is noteworthy that the levels of PRO-C3 did not increase from F3 to F4. The explanation for this finding is not clear and further mechanistic studies are required.

Previous reports have suggested that the pro-peptide of type III collagen ${ }^{22,36}$ can be used as a biomarker for NASH. However, we have shown that PRO-C3 is distinct from PIIINP in that it is a true marker of type III collagen formation and by extension, fibrogenesis ${ }^{22}$. We subsequently developed a novel PRO-C3-based fibrosis score for NAFLD patients and compared it to various composite serum based score systems that have been proposed and tested in NAFLD patients, namely APRI, FIB-4 and the NAFLD Fibrosis Score. The AUROCs for the various scores examined in this study, all performed similar to previous reports for the identification of advanced fibrosis ${ }^{27,37-39}$. In contrast, ADAPT was superior, as also in the multi-national validation cohort. Critically, ADAPT was robust at identifying patients with advanced fibrosis across different subpopulations (diabetics vs non-diabetics, NAFL vs $\mathrm{NASH}$, various age ranges and BMI categories), some of which have been shown to confound noninvasive algorithms ${ }^{40,41}$. The AUROC of ADAPT was maintained at $>0.80$ for all subpopulations, while the PPV and NPV remained consistent. From a management perspective, after the application of a derived cut-off value, ADAPT correctly classified $74 \%$ of patients without advanced fibrosis and $92 \%$ with advanced fibrosis. Cut-off values for APRI, FIB-4 and NAFLD Fibrosis Score were applied to our patients; similar to previous reports, we found that a large proportion of patients fell within an indeterminate zone ${ }^{37}$. FIB-4 and APRI showed reasonable performance at identifying patients without advanced fibrosis, but performed poorly at identifying patients with advanced fibrosis. Furthermore, the superiority of ADAPT is exemplified by its robust performance across various subpopulations (supplementary table 1 ) and by the substantially higher NPV. In contrast, the performance of FIB-4 has been demonstrated to be variable and is affected by confounders such as age. Additionally, unlike FIB-4, ADAPT is unburdened by the presence of an intermediate zone, which

This article is protected by copyright. All rights reserved. 
hinders its accuracy ${ }^{37,41-44}$. An advantage of PRO-C3 used alone or in combination as in ADAPT, is that it may stratify cirrhosis since the score is on a spectrum. This contrasts with FIB-4 or the NAFLD fibrosis score which are based on a dichotomous threshold. Hence, PRO-C3 based scores may have potential in patient monitoring over time, though this needs validation.

In contrast to the other non-invasive scores, ADAPT is distinct in that it combines PRO-C3 with important clinical and metabolic parameters associated with disease severity. Both increased age and the presence of diabetes are well-established risk factors for progressive liver disease and are easily discerned ${ }^{45}$. Similarly, platelet count is routinely measured and is strongly correlated with liver fibrosis and has been incorporated into multiple other non-invasive scoring systems ${ }^{10,12,14}$. A study by Mofrad et al has shown that the full spectrum of liver fibrosis stages can be found in patients presenting with liver enzymes in the normal range $^{8}$. In addition, liver enzymes are sensitive to age leading to false positive results. Thus, previous analysis has shown that FIB-4 (and likely also APRI and the NAFLD fibrosis score) cannot be universally applied without modification to all patient groups $^{41}$. The lack of inclusion of liver enzymes in ADAPT is thus a conspicuous advantage.

Non-invasive tests have been proposed as screening tools for detecting advanced liver fibrosis in the general population, where the prevalence of this outcome is low ${ }^{46}$. Score systems such as ADAPT, that exhibit a high specificity and NPV could provide a useful tool for clinicians as they reduce any uncertainty surrounding the diagnosis and the number of follow-up assessments required ${ }^{46}$. We propose that the ADAPT score could be used as such a screening tool within the general population to identify patients at risk of or with advanced fibrosis, such that interventions could be applied and progression to cirrhosis perhaps mitigated. However, further validation in non-referral cohorts and demonstration of the cost-effectiveness of using PRO-C3 based score systems is first required.

Our study has some limitations that must be acknowledged. We included well-characterized biopsied patients from centers with an interest in studying NAFLD, therefore referral bias cannot be ruled out. Biopsies were read by an independent pathologist at each participating center using a

This article is protected by copyright. All rights reserved. 
well-defined and standardized score system. In our hands, the kappa value for assessing the severity of fibrosis has previously been shown to be $\operatorname{good}^{47}$. As previously described by Ratziu et al, liver biopsy as a diagnostic tool has several limitations including sampling bias ${ }^{6}$. However, all non-invasive diagnostic tools for fibrosis assessment are benchmarked against the biopsy. Thus, the use of an imperfect reference standard may result in underperformance of the accuracy of non-invasive scores. Additionally, due to the nature of this cross-sectional study, we could not follow the clinical progress of patients; it would be of interest to investigate the relationship of score classification with patient outcome.

In conclusion, a biomarker score based on PRO-C3 and clinical variables (ADAPT) accurately predicts the presence or absence of advanced fibrosis in a NAFLD population. Thus, ADAPT could be useful for risk stratification and management. Further independent studies will be required to determine whether patients stratification using ADAPT followed by measurement of liver stiffness can replace the need for liver biopsy as a diagnostic standard in NAFLD.

\section{References}

1. Younossi, Z. et al. Global burden of NAFLD and NASH: trends, predictions, risk factors and prevention. Nat. Rev. Gastroenterol. Hepatol. (2017). doi:10.1038/nrgastro.2017.109

2. Angulo, P. et al. Liver Fibrosis, but No Other Histologic Features, Is Associated With Long-term Outcomes of Patients With Nonalcoholic Fatty Liver Disease. Gastroenterology 149, 389397.e10 (2015).

3. Ekstedt, M. et al. Fibrosis stage is the strongest predictor for disease-specific mortality in NAFLD after up to 33 years of follow-up. Hepatology 61, 1547-1554 (2015).

4. Younossi, Z. M. et al. Pathologic criteria for nonalcoholic steatohepatitis: Interprotocol agreement and ability to predict liver-related mortality. Hepatology 53, 1874-1882 (2011).

5. Hagström, H. et al. Fibrosis stage but not NASH predicts mortality and time to development of severe liver disease in biopsy-proven NAFLD. J. Hepatol. 67, 1265-1273 (2017).

6. Ratziu, V. et al. Sampling variability of liver biopsy in nonalcoholic fatty liver disease. Gastroenterology 128, 1898-906 (2005).

7. Regev, A. et al. Sampling error and intraobserver variation in liver biopsy in patients with chronic HCV infection. Am. J. Gastroenterol. 97, 2614-2618 (2002).

This article is protected by copyright. All rights reserved. 
8. Mofrad, P. et al. Clinical and histologic spectrum of nonalcoholic fatty liver disease associated with normal ALT values. Hepatology 37, 1286-1292 (2003).

9. Armstrong, M. J. et al. Severe asymptomatic non-alcoholic fatty liver disease in routine diabetes care; a multi-disciplinary team approach to diagnosis and management. QJM 107, 33-41 (2014).

10. Wai, C. T. et al. A simple noninvasive index can predict both significant fibrosis and cirrhosis in patients with chronic hepatitis C. Hepatology 38, 518-526 (2003).

11. Ratziu, V. et al. Liver fibrosis in overweight patients. Gastroenterology 118, 1117-23 (2000).

12. Sterling, R. K. et al. Development of a simple noninvasive index to predict significant fibrosis in patients with HIV/HCV coinfection. Hepatology 43, 1317-1325 (2006).

13. Forns, X. et al. Identification of chronic hepatitis $\mathrm{C}$ patients without hepatic fibrosis by a simple predictive model. Hepatology 36, 986-992 (2002).

14. Angulo, P. et al. The NAFLD fibrosis score: A noninvasive system that identifies liver fibrosis in patients with NAFLD. Hepatology 45, 846-854 (2007).

15. European Association for Study of Liver \& Asociacion Latinoamericana para el Estudio del Higado. EASL-ALEH Clinical Practice Guidelines: Non-invasive tests for evaluation of liver disease severity and prognosis. J. Hepatol. 63, 237-264 (2015).

16. Kleiner, D. E. et al. Design and validation of a histological scoring system for nonalcoholic fatty liver disease. Hepatology 41, 1313-1321 (2005).

17. Association for the Study of the Liver, E., Association for the Study of Diabetes, E. \& Association for the Study of Obesity, E. EASLâ€“EASDâ€"EASO Clinical Practice Guidelines for the management of non-alcoholic fatty liver disease. (2015). doi:10.1016/j.jhep.2015.11.004

18. Younossi, Z. M. et al. Nonalcoholic fatty liver disease: assessment of variability in pathologic interpretations. Mod. Pathol. 11, 560-5 (1998).

19. Fukusato, T. et al. Interobserver variation in the histopathological assessment of nonalcoholic steatohepatitis. Hepatol. Res. 33, 122-127 (2005).

20. Kazankov, K. et al. The macrophage activation marker SCD163 is associated with morphological disease stages in patients with non-alcoholic fatty liver disease. Liver Int. 36, 1549-1557 (2016).

21. Gawrieh, S., Knoedler, D. M., Saeian, K., Wallace, J. R. \& Komorowski, R. A. Effects of interventions on intra- and interobserver agreement on interpretation of nonalcoholic fatty liver disease histology. Ann. Diagn. Pathol. 15, 19-24 (2011).

22. Nielsen, M. J. et al. The neo-epitope specific PRO-C3 ELISA measures true formation of type III collagen associated with liver and muscle parameters. Am. J. Transl. Res. 5, 303-15 (2013).

23. Shah, A. G. et al. Comparison of Noninvasive Markers of Fibrosis in Patients With Nonalcoholic Fatty Liver Disease. Clin. Gastroenterol. Hepatol. 7, 1104-1112 (2009).

24. Shafer, G. A Mathematical Theory of Evidence. (Princeton University Press, 1976).

25. Lambert, J. et al. How to Measure the Diagnostic Accuracy of Noninvasive Liver Fibrosis Indices: The Area Under the ROC Curve Revisited. Clin. Chem. 54, 1372-1378 (2008).

This article is protected by copyright. All rights reserved. 
26. Obuchowski, N. A. Estimating and Comparing Diagnostic Tests' Accuracy When the Gold Standard Is Not Binary1. Acad. Radiol. 12, 1198-1204 (2005).

27. Poynard, T. et al. Standardization of ROC Curve Areas for Diagnostic Evaluation of Liver Fibrosis Markers Based on Prevalences of Fibrosis Stages. Clin. Chem. 53, 1615-1622 (2007).

28. Eslam, M. et al. FibroGENE: A gene-based model for staging liver fibrosis. J. Hepatol. 64, 390398 (2016).

29. Hanley, J. A. \& McNeil, B. J. A method of comparing the areas under receiver operating characteristic curves derived from the same cases. Radiology 148, 839-843 (1983).

30. Leeflang, M. M. G., Bossuyt, P. M. M. \& Irwig, L. Diagnostic test accuracy may vary with prevalence: implications for evidence-based diagnosis. J. Clin. Epidemiol. 62, 5-12 (2009).

31. Nielsen, M. J. et al. Markers of collagen remodeling detect clinically significant fibrosis in chronic hepatitis C patients. PLoS One 10, 1-12 (2015).

32. Nielsen, M. J. et al. Plasma Pro-C3 (N-terminal type III collagen propeptide) predicts fibrosis progression in patients with chronic hepatitis C. Liver Int. 35, 429-437 (2015).

33. Karsdal, M. A. et al. Fibrogenesis assessed by serological type III collagen formation identifies patients with progressive liver fibrosis and responders to a potential antifibrotic therapy. Am. J. Physiol. - Gastrointest. Liver Physiol. 311, G1009-G1017 (2016).

34. Harrison, S. et al. NGM282 improves fibrosis and NASH-related histology in 12 weeks in patients with biopsy-confirmed NASH, which is preceded by significant decreases in hepatic steatosis, liver transaminases and fibrosis markers at 6 weeks. J. Hepatol. 68, S65-S66 (2018).

35. Nielsen, M. J. et al. Fibrosis is not just fibrosis - basement membrane modelling and collagen metabolism differs between hepatitis B- and C-induced injury. Aliment. Pharmacol. Ther. 44, 1242-1252 (2016).

36. Tanwar, S. et al. Validation of terminal peptide of procollagen III for the detection and assessment of nonalcoholic steatohepatitis in patients with nonalcoholic fatty liver disease. Hepatology 57, 103-111 (2013).

37. Adams, L. A. et al. Complex non-invasive fibrosis models are more accurate than simple models in non-alcoholic fatty liver disease. J. Gastroenterol. Hepatol. 26, 1536-1543 (2011).

38. Dowman, J. K., Tomlinson, J. W. \& Newsome, P. N. Systematic review: the diagnosis and staging of non-alcoholic fatty liver disease and non-alcoholic steatohepatitis. Aliment. Pharmacol. Ther. 33, 525-40 (2011).

39. Shah, A. G. et al. Comparison of Noninvasive Markers of Fibrosis in Patients With Nonalcoholic Fatty Liver Disease. Clin. Gastroenterol. Hepatol. 7, 1104-1112 (2009).

40. Castéra, L. et al. Pitfalls of liver stiffness measurement: A 5-year prospective study of 13,369 examinations. Hepatology NA-NA (2010). doi:10.1002/hep.23425

41. McPherson, S. et al. Age as a Confounding Factor for the Accurate Non-Invasive Diagnosis of Advanced NAFLD Fibrosis. Am. J. Gastroenterol. 112, 740-751 (2017).

42. McPherson, S., Stewart, S. F., Henderson, E., Burt, A. D. \& Day, C. P. Simple non-invasive fibrosis scoring systems can reliably exclude advanced fibrosis in patients with non-alcoholic fatty liver disease. Gut 59, 1265-1269 (2010).

This article is protected by copyright. All rights reserved. 
43. Petta, S. et al. The combination of liver stiffness measurement and NAFLD fibrosis score improves the noninvasive diagnostic accuracy for severe liver fibrosis in patients with nonalcoholic fatty liver disease. Liver Int. 35, 1566-1573 (2015).

44. Xun, Y. H. et al. Suboptimal performance of simple noninvasive tests for advanced fibrosis in Chinese patients with nonalcoholic fatty liver disease. J. Dig. Dis. 13, 588-595 (2012).

45. Angulo, P., Keach, J. C., Batts, K. P. \& Lindor, K. D. Independent predictors of liver fibrosis in patients with nonalcoholic steatohepatitis. Hepatology 30, 1356-1362 (1999).

46. Mahady, S. E. et al. Diagnostic Accuracy of Noninvasive Fibrosis Scores in a Population of Individuals With a Low Prevalence of Fibrosis. Clin. Gastroenterol. Hepatol. 15, 1453-1460.e1 (2017).

47. Kazankov, K. et al. The macrophage activation marker SCD163 is associated with morphological disease stages in patients with non-alcoholic fatty liver disease. Liver Int. 36, 1549-1557 (2016).

Author names in bold designate shared co-first authorship

This article is protected by copyright. All rights reserved. 
Table 1. Characteristics of the total patient population

\begin{tabular}{|c|c|c|c|c|c|c|c|}
\hline & \multicolumn{3}{|c|}{ Derivation Cohort } & \multicolumn{3}{|c|}{ Validation Cohort } & \multirow{2}{*}{$\begin{array}{l}\text { Derivation cohort } \\
\text { vs Validation cohort } \\
\text { P-value }\end{array}$} \\
\hline & $\mathbf{n}$ & Mean & SD & $\mathbf{n}$ & Mean & SD & \\
\hline Age (Years) & 150 & 50.85 & 12.13 & 277 & 52.9 & 12.38 & ns \\
\hline Albumin (g/dL) & 148 & 4.403 & 5.54 & 277 & 4.07 & 0.40 & $<0.0001$ \\
\hline ALT (IU/L) & 148 & 77.01 & 50.48 & 278 & 69.58 & 58.43 & ns \\
\hline AST (IU/L) & 149 & 55.02 & 35.42 & 262 & 46.45 & 33.40 & 0.02 \\
\hline AST/ALT & 148 & 0.79 & 0.34 & 220 & 0.76 & 0.38 & ns \\
\hline $\mathrm{BMI}\left(\mathrm{kg} / \mathrm{m}^{2}\right)$ & 145 & 31.3 & 5.38 & 274 & 34.98 & 9.54 & $<0.0001$ \\
\hline Cholesterol (mmol/L) & 148 & 5.21 & 1.21 & 198 & 4.7 & 1.15 & 0.0001 \\
\hline Diabetic & 150 & $37.3 \%$ & & 281 & $37.4 \%$ & & ns \\
\hline Insulin (mIU/L) & 147 & 17.46 & 12.7 & 148 & 26.37 & 31.81 & 0.002 \\
\hline $\mathrm{FBS}$ (mmol/L) & 145 & 6.46 & 3.10 & 239 & 6.54 & 2.72 & ns \\
\hline $\begin{array}{l}\text { Fibrosis Score } \\
(0 / 1 / 2 / 3 / 4)\end{array}$ & \multicolumn{3}{|c|}{$42 / 48 / 27 / 25 / 8$} & \multicolumn{2}{|c|}{$90 / 87 / 37 / 44 / 21$} & & ns \\
\hline Gender (\% Female) & 150 & 50.7 & & 281 & 58 & & ns \\
\hline GGT (IU/L) & 148 & 128 & 141 & 256 & 112.57 & 7160.24 & ns \\
\hline $\mathrm{HDL}(\mathrm{mmol} / \mathrm{L})$ & 143 & 1.26 & 0.41 & 212 & 1.2 & 0.35 & 0.03 \\
\hline $\mathrm{LDL}(\mathrm{mmol} / \mathrm{L})$ & 140 & 3.07 & 0.99 & 179 & 2.78 & 1.56 & ns \\
\hline NASH & 55 & & & 127 & & & ns \\
\hline Platelets ( $\left.\times 10^{9} / \mathrm{L}\right)$ & 148 & 244.4 & 73.53 & 270 & 229.7 & 79.49 & ns \\
\hline PRO-C3 (ng/mL) & 150 & 20.92 & 15.48 & 279 & 19.93 & 18.04 & ns \\
\hline Triglycerides (mmol/L) & 149 & 2.03 & 1.60 & 263 & 1.97 & 1.40 & ns \\
\hline Waist/Hip ratio & 136 & 0.97 & 0.08 & 41 & 0.97 & 0.09 & ns \\
\hline
\end{tabular}

This article is protected by copyright. All rights reserved. 
Table 2 Multivariate logistic regression analyses

\begin{tabular}{lll}
\hline & Presence of Advanced Fibrosis & \\
\hline PRO-C3 Adjusted for & OR (95\% Cl) & P-value \\
\hline Unadjusted & 1.06 (1.03 to 1.09$)$ & 0.0003 \\
Age & 1.06 (1.03 to 1.09$)$ & 0.0003 \\
ALT & 1.07 (1.03 to 1.10$)$ & 0.0008 \\
AST & 1.05 (1.02 to 1.09$)$ & 0.0032 \\
Ballooning & 1.05 (1.02 to 1.08$)$ & 0.0015 \\
BMI & $1.06(1.02$ to 1.09$)$ & 0.0004 \\
Diabetes & $1.06(1.03$ to 1.09$)$ & 0.0002 \\
Gender & 1.06 (1.03 to 1.09$)$ & 0.0003 \\
GGT & 1.05 (1.01 to 1.08$)$ & 0.0044 \\
Lobular inflammation & 1.05 (1.02 to 1.08$)$ & 0.0008 \\
Platelets & 1.05 (1.02 to 1.09$)$ & 0.0019 \\
Fully Adjusted & 1.04 (1.01 to 1.07$)$ & 0.0078 \\
\hline Unadjusted and adjusted odds ratios (OR) with 95\% confidence intervals (Cl) for the increase in PRO- \\
C3
\end{tabular}

This article is protected by copyright. All rights reserved. 
Table 3 Predictors of Advanced Fibrosis

\begin{tabular}{|c|c|c|c|c|c|c|c|}
\hline & \multicolumn{3}{|c|}{$F=0-2$} & \multicolumn{4}{|c|}{$F=3-4$} \\
\hline & $\mathrm{n}$ & Mean & SD & $\mathrm{n}$ & Mean & SD & $P^{a}$ \\
\hline Age (Years) & 117 & 49.60 & 12.28 & 33 & 55.30 & 10.60 & 0.02 \\
\hline ALT (IU/L) & 116 & 74.31 & 45.10 & 32 & 86.81 & 66.44 & ns \\
\hline AST (IU/L) & 117 & 51.92 & 33.30 & 32 & 66.34 & 40.87 & 0.04 \\
\hline AST/ALT Ratio & 116 & 0.76 & 0.31 & 32 & 0.91 & 0.39 & 0.03 \\
\hline $\mathrm{BMI}\left(\mathrm{kg} / \mathrm{m}^{2}\right)$ & 113 & 30.99 & 5.46 & 32 & 32.37 & 4.98 & ns \\
\hline Diabetes & $29 \%$ & & & $67 \%$ & & & $0.0002^{b}$ \\
\hline $\mathrm{FBSL}(\mathrm{mmol} / \mathrm{L})$ & 114 & 6.49 & 3.38 & 31 & 6.37 & 1.70 & ns \\
\hline GGT (IU/L) & 116 & 111.53 & 124.14 & 32 & 187.78 & 180.08 & 0.006 \\
\hline $\mathrm{HDL}(\mathrm{mmol} / \mathrm{L})$ & 114 & 1.26 & 0.38 & 29 & 1.29 & 0.49 & ns \\
\hline Insulin (mIU/L) & 115 & 15.37 & 10.58 & 31 & 25.74 & 16.25 & $<0.0001$ \\
\hline $\mathrm{LDL}$ (mmol/L) & 112 & 3.19 & 0.96 & 28 & 2.57 & 0.96 & 0.002 \\
\hline Platelets $\left(\times 10^{9} / \mathrm{L}\right)$ & 115 & 254.17 & 66.14 & 33 & 210.45 & 87.89 & 0.002 \\
\hline PRO-C3 (ng/mL) & 117 & 17.87 & 13.10 & 33 & 31.72 & 18.42 & $<0.0001$ \\
\hline $\mathrm{TG}(\mathrm{mmol} / \mathrm{L})$ & 117 & 2.03 & 1.61 & 32 & 2.03 & 1.60 & ns \\
\hline Total cholesterol $(\mathrm{mmol} / \mathrm{l})$ & 116 & 5.29 & 1.12 & 32 & 4.89 & 1.46 & ns \\
\hline Waist $\backslash$ Hip ratio & 106 & 0.96 & 0.08 & 30 & 1.01 & 0.06 & 0.001 \\
\hline
\end{tabular}

Univariate analysis of variables to identify potential predictors of advanced fibrosis. ${ }^{\text {a }}$ T-test was assessed to test for significant differences within continuous variables and ${ }^{\mathrm{b}}$ Fisher's exact test was used for categorical variables

This article is protected by copyright. All rights reserved. 
Table 4

\begin{tabular}{|c|c|c|c|c|c|c|c|c|}
\hline \multirow{3}{*}{$\begin{array}{l}\text { Non-invasive test } \\
\text { APRI }\end{array}$} & \multicolumn{4}{|c|}{ Derivation Cohort } & \multicolumn{4}{|c|}{ Validation Cohort } \\
\hline & AUROC & AdjAUROCS & & $95 \% \mathrm{Cl}$ & AUROC & AdjAUROC & SD & $95 \% \mathrm{Cl}$ \\
\hline & 0.73 & 0.76 & 0.05 & 0.65 to 0.80 & 0.78 & 0.80 & 0.03 & 0.73 to 0.83 \\
\hline FIB-4 & 0.78 & 0.81 & 0.05 & 0.70 to 0.84 & 0.85 & 0.87 & 0.02 & 0.80 to 0.89 \\
\hline NAFLD Fibrosis Score & 0.78 & 0.82 & 0.05 & 0.71 to 0.85 & 0.79 & 0.81 & 0.03 & 0.74 to 0.84 \\
\hline PRO-C3 & 0.81 & 0.85 & 0.04 & 0.74 to 0.87 & 0.83 & 0.84 & 0.03 & 0.78 to 0.87 \\
\hline ADAPT & 0.86 & 0.89 & 0.04 & 0.79 to 0.91 & 0.87 & 0.89 & 0.02 & 0.83 to 0.91 \\
\hline
\end{tabular}

Table 5

\begin{tabular}{lllllll}
\hline & F0-2 & \multicolumn{5}{l}{ F3-4 } \\
\hline & $\begin{array}{llllll}\text { Correctly } \\
\text { Identified }\end{array}$ & $\begin{array}{l}\text { Indeterminate } \\
\text { Incorrectly }\end{array}$ & $\begin{array}{l}\text { Correctly } \\
\text { Identified }\end{array}$ & $\begin{array}{l}\text { Identified } \\
\text { Indeterminate }\end{array}$ & Identified \\
\hline$\Sigma$ & 216 & & 65 & & \\
APRI & 145 & 63 & 8 & 10 & 36 & 19 \\
FIB-4 & 147 & 54 & 15 & 30 & 25 & 10 \\
NFS & 91 & 100 & 25 & 33 & 25 & 7 \\
ADAPT & 158 & - & 58 & 60 & - & 5 \\
\hline
\end{tabular}

Number of patients correctly, incorrectly or indeterminately classified by the various noninvasive scores

This article is protected by copyright. All rights reserved. 

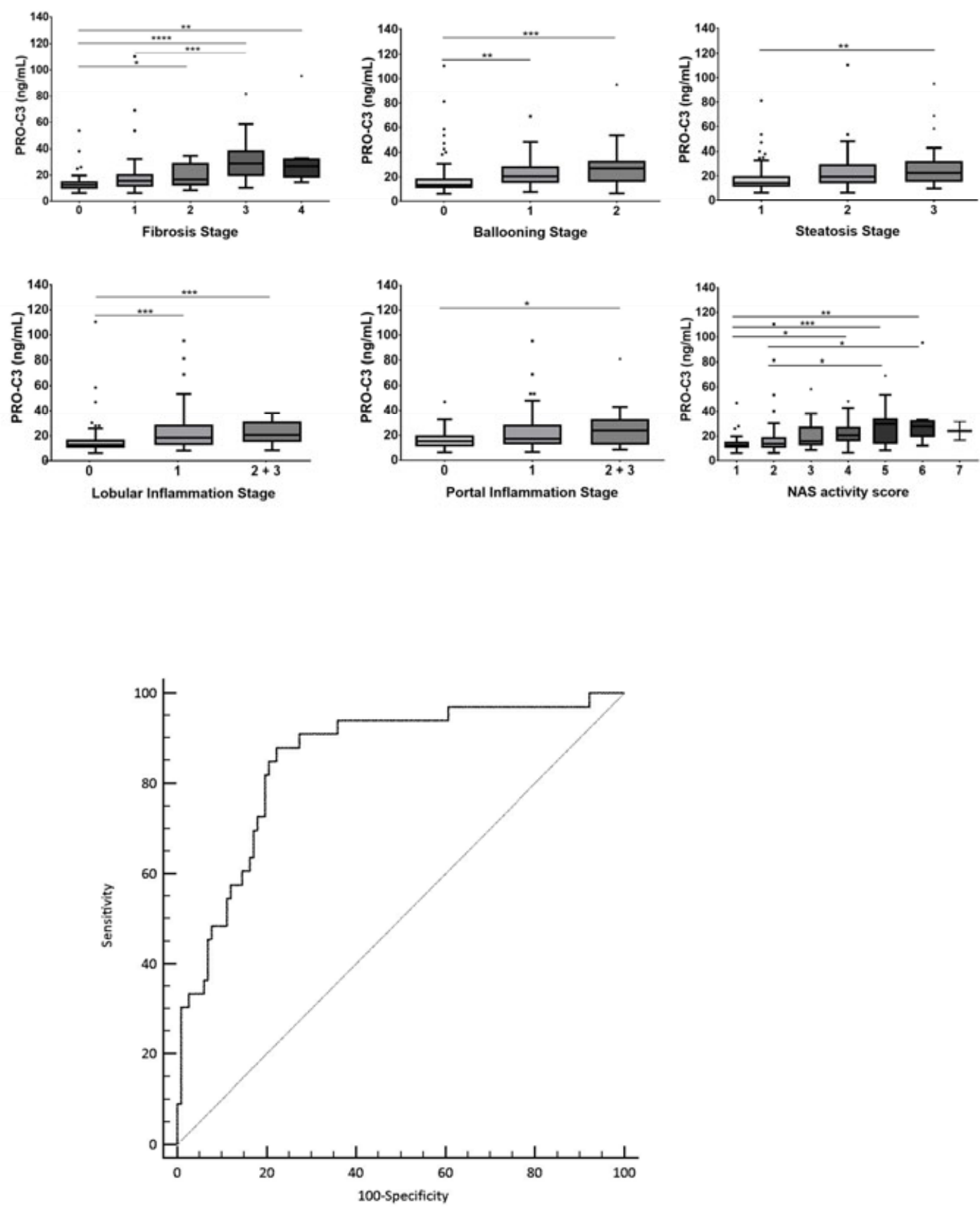

This article is protected by copyright. All rights reserved. 


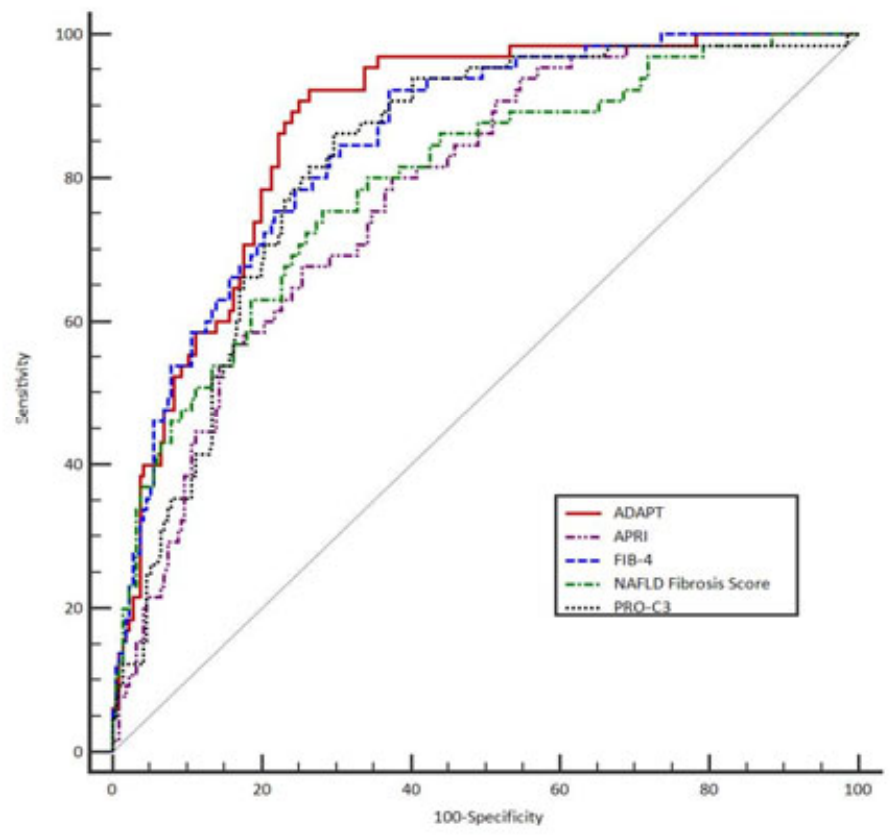

This article is protected by copyright. All rights reserved. 